\title{
Spontaneous intracerebral haemorrhage in patients suspected of multiple sclerosis
}

\author{
I. F. ABROMS ${ }^{1}$, L. YESSAYAN, J. SHILlito, AND C. F. BARLOW \\ From the Services of Neurology and Neurosurgery, Children's Hospital Medical Center, the Peter Bent Brigham \\ Hospital, and the Departments of Neurology and Surgery, Harvard Medical School, Boston, Mass., U.S.A.
}

SUMMARY Two cases of spontaneous intracerebral haemorrhage in adolescent girls suspected of having multiple sclerosis are reported. Surgical evacuation of haematomas in the left thalamus and left side of the pons, respectively, was performed with excellent recovery in both cases. Some clinical and pathological aspects of small arteriovenous malformations are discussed and the pertinent literature reviewed.

The purpose of this report is to describe two cases of spontaneous intracerebral haemorrhage, both in adolescent girls, in which the clinical diagnosis was initially thought to be multiple sclerosis. Early in the course, the cerebrospinal fluid (CSF) was normal and later cerebral arteriograms and air-studies revealed mass lesions in the thalamus and pons respectively. Both patients improved considerably after surgical evacuation of haematomas at these sites. Neither case had evidence of hypertension, blood disease, arteritis, neoplasm, or mycotic aneurysm. The cerebral arteriograms were negative for berry aneurysm and large arteriovenous malformation. No history of head trauma was obtained.

In recent years, Margolis, Odom, Woodhall, and Bloor (1951) and Russell (1954) have stressed the role of small arteriovenous malformations (AVM) in the production of spontaneous intracerebral haematomas in young adults and children. For several reasons (vide infra) we think that the intracerebral haematomas in our cases were due to bleeds from so-called 'cryptic' AVM (Crawford and Russell, 1956; McCormick and Nofzinger, 1966). The striking feature of both clinical presentations was the tendency for remissions and exacerbations which initially suggested multiple sclerosis as the diagnosis.

\section{CASE 1}

L.S. (CHMC no. 57-06-35) was a 16 year old, right-handed high school student in 1964. Her birth history and post-

\footnotetext{
${ }^{1}$ Fellow of the United Cerebral Palsy Foundation, Inc. Supported in part by the Children's Hospital Medical Center Mental Retardation and Human Development Research Program (HD 03-0773).
}

natal development had been normal; she had had no past neurological illnesses. Her parents and four siblings were well.

In April 1964, she developed mild occipital headache and the next day had weakness and a numb feeling in her right side. She also complained of numbness of her left cheek and gums, diplopia on looking towards the left, and some difficulty with hearing on that side. The persistence of the right-sided weakness during the ensuing week resulted in her admission to the Children's Hospital Medical Center, Boston.

Initial examination revealed a slender girl with normal vital signs and systemic examination; no cranial bruit was heard. Her mental status was normal and no dysphasia noted. Examination of her optic fundi, visual fields, and pupillary reactions were normal. On looking towards the left, horizontal nystagmus and diplopia were present, yet ocular movements had a full range. The left corneal reflex was depressed and sensation to touch and pinprick was decreased over the left forehead and cheek. Weakness of the facial muscles on the right was noted. Mild leftsided nerve deafness was confirmed by audiometry; rapid auditory fatigue and an absence of loudness recruitment were also found. She tended to fall to the right on walking and had a poor arm swing on that side; ankle clonus, hyperreflexia, and an extensor plantar response were found on the right side. Finger-to-nose and heel-to-shin testing revealed intention tremors bilaterally, but these were worse on the left side, similarly, with rapid alternating movements of the limbs. There was decreased sensation to pin, touch, and temperature over the right arm, trunk, and leg.

Initial studies included a normal haemogram, urinalysis and radiographs of the skull; an electroencephalogram showed some left anterior quadrant slowing. A lumbar tap revealed an opening pressure of $50 \mathrm{~mm}$ CSF. The fluid was clear and colourless and had a cell count of 2 monocytes/cu. mm.; the protein concentration was $32 \mathrm{mg} / 100 \mathrm{ml}$., and a colloidal gold curve was 0000000000 . 
Within six days of admission, she was much improved and on discharge on the 12th day only mild right-sided weakness and slight intention tremor of both upper extremities were still present. This illness was regarded as the initial attack of multiple sclerosis in this girl.

Two months later she was bowling, swimming, and dancing. On a follow-up clinic evaluation her hearing was normal and no right-sided motor weakness was noted.

Fourteen months (June 1965) after her first symptoms, she was found to be using her right arm less well and hyperreflexia was again present on that side. In October 1965 , she complained of numbness and weakness of the right leg. but six weeks later this was improved. She graduated from high school the following summer with honours.

Her next admission was in October 1966. For six weeks she had complained of occipital headaches, slurring of speech, and difficulty with fine movements of the right hand.

Her vital signs, mental status, and systemic examination were normal. Her pupils were equal in size, but the left responded poorly to light. There was a scanning dysarthria with poor movements of the palate and tongue. Slight distal upper extremity weakness, an increase in resistance to passive movement, hyperreflexia, and an extensor plantar response were found on the right side. The fingerto-nose and heel-to-shin tests and rapid alternating movements were abnormal on the right. There was decreased appreciation of position and vibration sense in the right lower extremity, while pin, touch, and temperature testing were normal.

Laboratory tests were again normal. The spinal fluid opening pressure was $135 \mathrm{~mm}$ CSF; the fluid was colourless and the cell count was 18 red blood cells and 1 monocyte/cu. $\mathrm{mm}$. The protein was $34 \mathrm{mg} / 100 \mathrm{ml}$., and the gold sol curve was normal. The patient was discharged four days later with the right-sided weakness and incoordination improved, but still present.

Within three days she was readmitted because of lethargy and an increase in her right-sided weakness. Her general examination and vital signs were unchanged. She was alert and orientated, but she was noted to have a blandness of affect and slowed speech. The cranial nerve signs, right-sided weakness, intention tremor, and sensory signs were the same as on the first day of her previous admission. Normal laboratory studies included a urinalysis, a full haemogram, sedimentation rate, BUN, electrolytes, liver function tests, heterophile antibody test, two LE preparations, and Hinton test. $A{ }^{99}$ technetium brain scan was also normal.

Multiple sclerosis was thought to be the most likely diagnosis and a course of ACTH was started and continued for two weeks.

Three days after admission the patient became disorientated to time and place; she had diplopia on forward gaze with vertical nystagmus, and the right-sided weakness and intention tremor were worse. She gradually improved, however, over the ensuing two weeks.

A spinal tap at that time revealed unexpected xanthochromia. The opening pressure was $145 \mathrm{~mm} \mathrm{CSF}$; there were no cells. A CSF bilirubin was $2 \cdot 2 \mathrm{mg} / 100 \mathrm{ml}$. One week later a repeat spinal fluid examination revealed an opening pressure of $250 \mathrm{~mm} \mathrm{CSF}$ and increased xanthochromia; a cell count revealed four red blood cells and two monocytes/cu. mm.; protein was $44 \mathrm{mg} / 100 \mathrm{ml}$. with $2.5 \mathrm{mg} / 100 \mathrm{ml}$. gamma globulin; the gold sol curve was of 0000000000 . An electroencephalogram showed paroxysmal delta slowing over the left hemisphere and an echo- $T$ encephalogram suggested a mass lesion in the left thalamic region.

A left carotid and right brachial arteriogram showed lateral displacement of the left internal cerebral vein with stretching and downward displacement of the basal vein of Rosenthal by a deep left parasagittal mass. Before $\overrightarrow{\mathcal{O}}$ surgery a pneumoencephalogram was done. This revealed displacement of the middle and posterior portions of the third ventricle from left to right, up to $7 \mathrm{~mm}$ (Fig. 1). The anterior part of the third ventricle was slightly dilated and the lateral ventricles moderately dilated. The aqueduct and fourth ventricle were normal.

A left parietal craniotomy was performed and the left thalamus was approached transcortically through the parietal sensory association area. Good exposure of the posterior thalamus was obtained at the atrium of the $\stackrel{\sigma}{\sigma}$ lateral ventricle, facilitated by the ventricular enlarge- $\overline{\bar{J}}$ ment. The overlying choroid plexus was removed. A brain needle was passed into the enlarged thalamus and a cavity $\omega$ was encountered $\frac{1}{2}$ centimetre from the ventricular surfaceo

FIG. 1. (L.S.). Pneumoencephalogram: brow-up frontal ${ }^{N}$ view. The third ventricle is maximally displaced posteriorly (upper arrow) and approaching the midline anteriorly (lower arrow). There is moderate lateral ventricular dilata- $\omega$ tion. 
It was more than $2 \mathrm{~cm}$ in diameter and contained old blood. Biopsy of its yellow wall revealed cell necrosis and haemosiderin-filled macrophages, but no abnormal blood vessels or malignant cells. The cavity was irrigated and the craniotomy closed.

The patient did well postoperatively and within two weeks the intracranial pressure returned to normal. On discharge three weeks after surgery she still had slight dysarthria and a mild right hemiparesis.

When seen in the clinic four months later, she had mild weakness of dorsiflexion of the right foot and loss of fine motor skills of the right hand. Coarse horizontal nystagmus and mild diplopia were present on left lateral gaze.

Her hemiparesis continued to improve and in June 1968 , she was married.

In December of that year, she hit her forehead on a door and within a few hours had vertigo and marked diplopia on left lateral gaze. She also developed frontal headache, right sided incoordination and numbness which lasted about three weeks. However, the diplopia and occasional vertigo continued to be troublesome. When seen in February 1969, she was six months pregnant. Her neurological examination revealed a slight head tilt towards the right; a curious periodic conjugate clockwise rotation of the eyes in all directions of gaze, and mild right-sided weakness and ataxia.

In May 1969, the patient had an uneventful delivery of a full-term infant. When last seen in March 1970, the patient still complained of periodic involuntary clockwise rotations of the eyes lasting about 10 minutes, which were gradually improving in that they tended to occur less frequently.

\section{CASE 2}

G.M. (PBBH no. 8-65-80) was a 15 year old, righthanded, high school student who entered the Peter Bent Brigham Hospital, Boston, on 25 July 1967. Her past medical history and family history were unremarkable. On admission her chief complaint was diplopia. She had been perfectly well until three and a half weeks before admission, when she began to experience episodes of vertigo associated with mild right frontal headache. This was worse at the onset, but not incapacitating, and gradually improved over the next 10 days. Two weeks before admission, while painting the back porch, she became aware of numbness in the right thumb and, later, in the right hand, which was attributed to the task she was performing. Four hours later, she felt the numbness had spread to the entire right arm and leg. She also realized that her gait had become unsteady and her coordination poor. Although she denied weakness, she began to use the left arm more. A neurologist was consulted, who noted the right-sided sensory change and the slightly ataxic gait as the only findings. The cranial nerves, including the optic discs, showed no abnormalities, and her speech was normal. The clinical impression then was that this represented a probable initial attack of multiple sclerosis, and the patient was started on vitamin B injections. Again, she showed some questionable improvement over the next few days, but this was less convincing compared with the previous week. One week before admission, she noticed diplopia on left lateral gaze, which persisted until admission. She denied any history of head trauma, seizures, dysarthria, or any previous similar episodes.

Examination revealed a well-developed, alert, attractive girl in no acute distress. The vital signs were normal. There was one café-au-lait spot measuring $4.0 \times 1.0 \mathrm{~cm}$, over the right side of the chest anteriorly, but otherwise the general physical examination was entirely normal. No cranial bruits were heard. Her neurological examination showed an essentially normal mental status. Visual fields and fundi were normal bilaterally. There was a left facial weakness and taste was lost on the anterior two-thirds of the tongue on the left. Vertical and horizontal nystagmus on right lateral gaze was observed. The gag reflex was slightly diminished. There was mild right hemiparesis; rapid alternating movements were done poorly on the right. She had an intention tremor on finger-to-nose and heel-to-shin testing on the right. Her gait was unsteady, with minimal dragging of her right leg. Deep tendon reflexes were increased, with an equivocal plantar response on the right side. Sensory examination showed diminished sensation to all modalities on the right side.

The initial investigations included a normal urinalysis, haemogram, sedimentation rate, electrolytes, BUN, blood glucose, liver function tests, and LE preparation. Radiographs of the skull, an electroencephalogram, and a ${ }^{99}$ technetium brain scan were normal. Lumbar puncture showed an opening pressure of $170 \mathrm{~mm}$ CSF. The CSF was questionably minimally xanthochromic, with a protein of $58 \mathrm{mg} / 100 \mathrm{ml}$, gamma globulin fraction of $22 \%$, and a normal colloidal gold curve. There were no cells.

During initial observation in the hospital, she showed worsening of her hemiparesis and facial weakness and developed bilateral internuclear ophthalmoplegia which was more complete on the left side. She was then started on a course of intravenous ACTH, with the assumption that this left pontine lesion probably represented her first attack of multiple sclerosis. After the first week of therapy, some observers felt there was a questionable improvement, which was not convincing to all. Therefore, after a two week course, because of lack of any significant improvement, a pneumoencephalogram was done. This revealed a left pontine mass elevating the fourth ventricle and displacing to the right (Fig. 2). Because of the asymmetry of the lesion, a posterior fossa exploration was scheduled as it was felt this was atypical for the usual pontine glioma.

On retracting the cerebellar tonsils upward, a mass could be seen protruding from the left half of the floor of the fourth ventricle, displacing it to the right. Discolouration suggested that this might be cystic and, indeed, old chocolate coloured blood was drained from this area, which reduced the mass completely. The contents were estimated as less than $10 \mathrm{ml}$. No vascular malformation could be seen grossly; the cyst wall was quite smooth, and a biopsy of it showed only clotted blood and haemosiderin-containing macrophages. No malignant cells or abnormal blood vessels were found.

Immediately after surgery, and on the first postoperative day, the patient was described as being fully alert and orientated, moving all four limbs. The next day she 


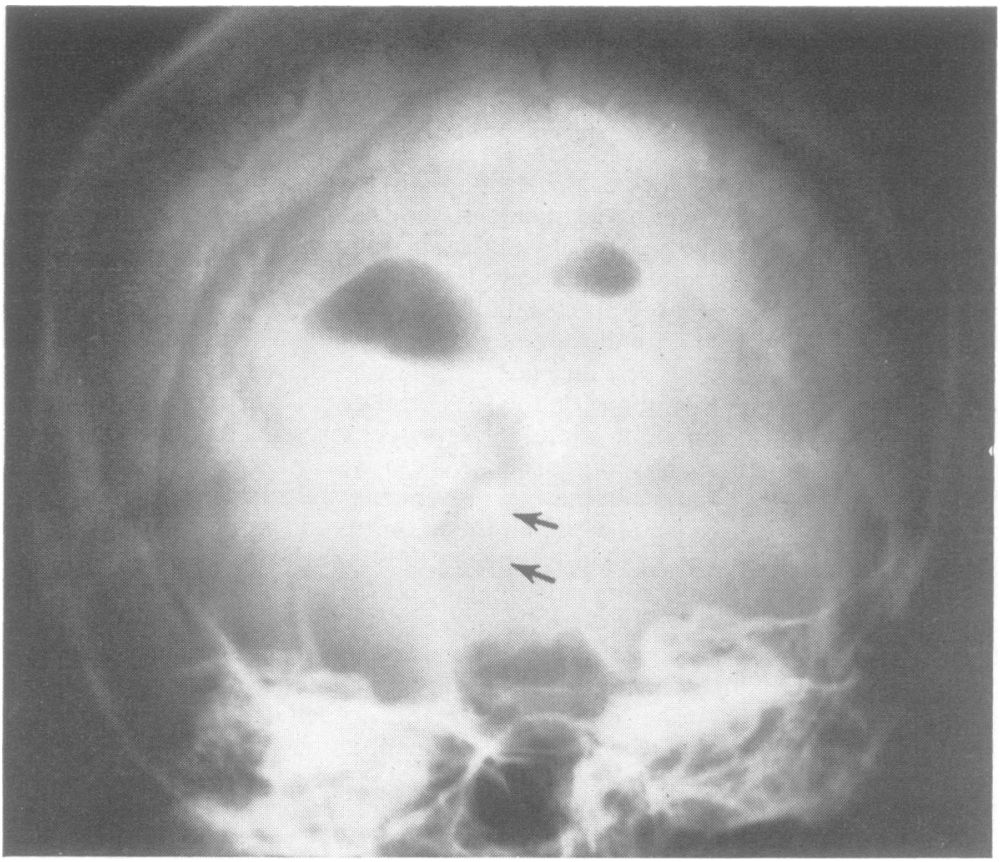

FIG. 2. (G.M.).

Pneumoencephalogram: upright filling view, anteroposterior projection. The fourth ventricle is seen to be displaced to the right and its foor asymmetrically elevated, suggesting an intrinsic mass. This is not characteristic of the usual inoperable pontine glioma. developed a persistent tachycardia of 140 to 150 per minute. On the third morning, she was difficult to arouse and her right hemiparesis had worsened. Repeat lumbar puncture showed an opening pressure of $400 \mathrm{~mm} \mathrm{CSF}$, with bloody fluid. She underwent reexploration of the posterior fossa, with evacuation of a subdural and extradural blood clot and continuous ventricular drainage was installed as a precaution. In the recovery room, she was described as being awake, opening her eyes, moving her tongue, her right side and her right hand. The next day she was unable to move all four extremities except for the right hallux, and had a decerebrate response on the left side to painful stimuli. She was thought to be aware of her environment and comprehending, as she was able to move the right toe appropriately to command. The rest of her five week hospital stay was essentially uncomplicated, with very gradual but steady partial recovery of function, particularly in the lower extremities. On the 24 th postoperative day, she started to walk with assistance and regain her speech. Five weeks postoperatively, her speech was dysarthric, but no indication of expressive language disturbance was found. She had no evidence of auditory agnosia, but had difficulty in dealing with visual language symbols, with impairment of silent and oral reading skills, and also with arithmetic. She was discharged to a rehabilitation centre for active physical, educational, and speech therapy.

The patient was readmitted one year later for reevaluation and vertebral angiography. After spending the year at the Vermont Rehabilitation Center, she returned to her regular high school. She had no complaints. Her speech was clear, with only occasional slur- ring of a few words. Mental status was interpreted being normal. She claimed to be totally amnesic for th month after surgery. The residual cranial nerve sign 8 included diminished sensation over the forehead on the left and marked facial weakness on that side whis included the frontalis and the orbicularis oculi muscles: Bilateral horizontal and vertical nystagmus was noted. She dragged her right leg slightly on walking and had some difficulty with tandem gait. She had mild limb ataxia bilaterally. The deep tendon reflexes were more active on the right, with a probable right extensor plantar response. There was also slight decrease to pain and difficulty with position sense on the right side.

A vertebral angiogram, done by the femoral route, showed no abnormalities and no vascular malformation in the pontine area.

When last seen, in March 1970, she was doing well as a senior in high school.

\section{DISCUSSION}

An interesting aspect of these cases has been the 8 subacute or chronic course, with apparent remissions. Both girls were diagnosed as having multiple 0 sclerosis at early stages of their illnesses, and were treated with ACTH. The eventual appearance of increased spinal fluid pressure and xanthochromia led in case 1 to radiological contrast procedures $\sigma$ which revealed a mass lesion. In case 2 , a pneumo- $N$ encephalogram was done to exclude a pontine tumour $N$ because of worsening of the brain-stem signs. The $\frac{\sigma}{\sigma}$ 
asymmetry of the pontine signs clinically and radiographically prompted an exploration of the posterior fossa.

Although there is no evidence that the mass lesions in these cases were neoplastic, it is important to emphasize that brain-stem tumours can produce relapsing and remitting symptoms and signs which clinically may simulate multiple sclerosis. Sarkari and Bickerstaff (1969) recently reported four such cases in children and young adults with remitting and relapsing clinical courses of up to 12 months. Three of these cases had astrocytomas of the brain-stem at necropsy, while the fourth case had a vascular tumour at the cerebello-pontine angle.

There are several reports of large AVM presenting with a remitting course (Martin, 1951; Hierons, 1953; Teilmann, 1953; Verbiest, 1960-61); this clinical picture is rarer with small AVM. The case of Lindheimer and Barrows (1962), in which recurrent episodes of pontine dysfunction occurred in a 43 year old woman over an 18 month period, most resembles ours. She died in her third attack and a gross pontine haemorrhage secondary to a venous angioma was found at necropsy. Initially, CSF studies, vertebral angiography and a pneumoencephalogram were all normal. Late in the course, raised intracranial pressure and CSF xanthochromia suggested an AVM. In the third case reported by Margolis et al. (1951) and the sixth case in Crawford and Russell's series (1956), there was clinical as well as pathological evidence of repeated intracerebral hemispheral bleeding from small malformed vessels. These cases occurred in children, aged 7 and 12 years respectively.

It is possible that small recurrent bleeds from an AVM could account for the remitting clinical course in our cases, although no definite pathological proof is available.

In an extensive review of pontine haemangiomas by Teilmann (1953) only four of 45 cases were in persons under 18 years. Of interest is the report of a pontine haemorrhage from a cavernous haemangioma in a girl aged 2 years (Grotts, 1950).

In case 2 , all the neurological signs could be explained by a single left-sided pontine lesion. In case 1 , the anatomical localization was more difficult. Initially, the left-sided eye signs and hearing impairment suggested a left ponto-medullary lesion. The exacerbation of diplopia to the left after minor head trauma two years after her thalamic operation further attests to the presence of a lesion in this location. The early bilateral cerebellar signs could not be explained by this lesion. The basis for these signs remains undefined, but one could speculate that they are due to a similar pathology which led to a bleed into the left thalamus. Of 15 cases of multiple telangiectasis of the brain reviewed by Michael and Levin (1936), three had lesions in both the diencephalon and the pons.

The possibility of coexisting multiple sclerosis, particularly in case 1 , cannot be ruled out. However, repeated CSF examinations yielded normal proteins, gamma globulin concentration, and colloidal gold curves in case 1 ; in case 2 , the protein level and gamma globulin level were elevated on one occasion. Spontaneous intracerebral haemorrhage occurring in subacute or chronic cases of multiple sclerosis is most unusual (McAlpine, Compston, and Lumsden, 1955). Evidence of haemorrhage into the CSF is, of course, a feature of acute haemorrhagic leucoencephalitis (Hurst, 1941). The clinical picture in this syndrome is more fulminant than that of our cases, but subacute and recurrent forms have been described (Adams, 1959; Behan, Geschwind, Lamarche, Lisak, and Kies, 1968).

The common sites in the brain of single small AVM in recent reports have included subependymal areas (Bailey and Woodard, 1959; Matson, 1969); the brain surface and in the depths of sulci (Russell, 1954; Krayenbühl and Siebenmann, 1965); and the basis pontis and cerebellum (Bergman, 1950; Teilmann, 1953, Logue and Monckton, 1956; Verbiest, 1960-61). Twenty of 461 cases of intracranial haemorrhages reviewed by Russell (1954) were due to 'cryptic' AVM. Half of these were located in the cerebral convexities, four were 'central cerebral', and six were in the cerebellum. All 20 cases were persons under 40 years and 15 were under 20 years. McCormick and Nofzinger (1966) found 161 of 308 small AVM were supratentorial; 85 of the remainder were in the brain-stem, 44 in the cerebellum, and 18 in the spinal cord.

Recovery from haemorrhage due to small AVM situated deep in the cerebral hemispheres and pons is rare (Russell, 1954). It is important to stress that even these relatively inaccessible intracerebral AVM (Carton and Hickey, 1955) and haematomas are remediable and can result in excellent recovery. The subependymal locus of these lesions may permit surgical access through the lateral ventricle, as in case 1.

We would like to thank Dr. H. Richard Tyler and Dr. Floyd H. Gilles for their helpful criticism; Dr. Roy Strand and Dr. J. Stovring; and Miss E. Luther for her secretarial assistance in the preparation of this paper.

\section{REFERENCES}

Adams, R. D. (1959). A comparison of the morphology of the human demyelinative diseases and experimental 'allergic' encephalomyelitis. In 'Allergic' Encephalomyelitis. Edited by M. W. Kies and E. C. Alvord. p. 183. Thomas: Illinois. 
Bailey, O. T., and Woodard, J. S. (1959). Small vascular malformations of the brain: their relationship to unexpected death, hydrocephalus, and mental deficiency. J. Neuropath. exp. Neurol., 18, 108-113.

Behan, P. O., Geschwind, N., Lamarche, J. B., Lisak, R. P., and Kies, M. W. (1968). Delayed hypersensitivity to encephalitogenic protein in disseminated encephalomyelitis. Lancet, 2, 1009-1012.

Bergman, P. S. (1950). Hemangioma of the pons. Case report and review of the literature. J. Mt Sinai Hosp., 17, 119-131.

Carton, C. A., and Hickey, W. C. (1955). Arteriovenous malformation of the head of the caudate nucleus. $J$. Neurosurg., 12, 414-418.

Crawford, J. V., and Russell, D. S. (1956). Cryptic arteriovenous and venous hamartomas of the brain. J. Neurol. Neurosurg. Psychiat., 19, 1-11.

Grotts, B. F. (1950). Spontaneous hemorrhage from a hemangioma of the pons. J. Pediat., 37, 98-101.

Hierons, R. (1953). Brain-stem angioma confirmed by arteriography. Relapsing symptoms and signs strongly suggestive of disseminated sclerosis. Proc. roy. Soc. Med., 46, 195-196.

Hurst, E. W. (1941). Acute hemorrhagic leucoencephalitis: a previously undefined entity. Med.J. Aust., 2, 1-6.

Krayenbühl, H., and Siebenmann, R. (1965). Small vascular malformations as a cause of primary intracerebral hemorrhage. J. Neurosurg., 22, 7-20.

Lindheimer, J. H., and Barrows, H. S. (1962). Recurrent pontine hemorrhage incident to venous angioma of the pons. Bull. Los Angeles neurol. Soc., 27, 128-133.
Logue, V., and Monckton, G. (1954). Posterior fossa angiomas. A clinical presentation of nine cases. Brain, 77, 252-273.

Margolis, G., Odom, G. L., Woodhall, B., and Bloor, B. M. (1951). The role of small angiomatous malformations in the production of intracerebral hematomas. J. Neurosurg., 8, 564-575.

Martin, J. P. (1951). Discussion on some less common cerebrovascular diseases. Angiomatous malformations. Proc. roy. Soc. Med., 44, 845-847.

Matson, D. D. (1969). Neurosurgery of Infancy and Childhood. 2nd edn. pp. 749-772. Thomas: Illinois.

McAlpine, D., Compston, N. D., and Lumsden, C. E. (1955). Multiple Sclerosis, p. 230. Livingstone: Edinburgh.

McCormick, W. F., and Nofzinger, J. D. (1966). 'Cryptic' vascular malformations of the central nervous system. $J$. Neurosurg., 24, 865-875.

Michael, J. C., and Levin, P. M. (1936). Multiple telangiectases of the brain. A discussion of hereditary factors in their development. Arch. Neurol. Psychiat., (Chic.) 36, 514-529.

Russell, D. S. (1954). The pathology of spontaneous intracranial hemorrhage. Proc. roy. Soc. Med., 47, 689-693.

Sarkari, N. B. S., and Bickerstaff, E. R. (1969). Relapses and remissions in brain stem tumours. Brit. med. J., 2, 21-23.

Teilmann, K. (1953). Hemangiomas of the pons. Arch. Neurol. Psychiat. (Chic.), 69, 208-223.

Verbiest, H. (1961). Arteriovenous aneurysms of the posterior fossa; analysis of six cases. Acta Neurochir. (Wien), 9 , 171-195. 